\title{
Human CRISPR
}

\section{A humanized version of the RNA-targeting Cas13 can regulate RNA abundance and translation.}

( ne of the main questions driving the research in the lab of Bryan Dickinson, at the University of Chicago, is how RNA modifications regulate gene expression. From their experience using CRISPR-Cas13 to engineer some of these modifications, the team realized two things. First, targeting RNA for therapy has substantial clinical potential. "The treatment is transient, which allows you to target multiple genes with temporal control," Dickinson explains. Second, there are challenges with a system that uses the large bacterial protein Cas13 to target RNA. Dickinson points out that this makes cloning and delivery harder, and that antibodies are likely to emerge quickly if the protein is chronically administered.

Their goal was to replace Cas 13 with a human protein. Graduate student Simone Rauch took on the project, and Dickinson gives her full credit for having the bravery and creativity to pursue something that many doubted would ever work.

Rauch needed four parts for a successful targeting system: a guide RNA (gRNA) complementary to the target RNA, a protein that would bind and stabilize the singlestranded gRNA prior to target binding, an effector protein that would introduce the desired changes, and, at the core of it all, a protein that would link all the components and keep them together.

The first rendition of their CIRTS (CRISPR-Cas-inspired RNA-targeting system) featured the nuclease domain of a human nonsense-mediated mRNA decay factor and reduced the mRNA and protein levels of the targeted luciferase reporter. Encouraged, the researchers then began to swap out different parts of CIRTS. They replaced the nuclease effector with a reader for the abundant $\mathrm{m}^{6} \mathrm{~A}$ modification on RNA that recruits the translation machinery and another $\mathrm{m}^{6} \mathrm{~A}$ reader that induces RNA degradation. The luciferase levels went up and down, respectively, as expected. When the team used the adenosine deaminase ADAR as an effector, they could trigger an A-to-I edit, which transformed a dead luciferase with a stop codon in its reading frame into an active protein.

CIRTS also proved effective when targeting endogenous transcripts. "To me, it was surprising that it worked so well," says Dickinson. "We don't exactly know how everything lines up when it binds to RNA. We screened and tested ideas. Once we got the first CIRTS to work, expanding it became much simpler."

There are some limitations to any RNA-targeting system. One can target only accessible sites, and computational predictions for such sites on RNA are only just becoming available. Dickinson reckons that the sites open to Cas 13 will also work for CIRTS and allow the design of gRNAs. The other question, of course, is where one wants to target an effector to achieve the best result. For each CIRTS, Dickinson envisions a Venn diagram of the sites that can be targeted and the physical requirements of where each effector should be placed. He thinks CIRTS will help elucidate some of these requirements and show for a given effector the differences in, for example, targeting it to the $5^{\prime}$ UTR, the coding region, or the $3^{\prime}$ UTR.

Dickinson is cautious to not oversell the approach. "It is not entirely human-based yet. The junctions between the proteins could still be problematic." He sees it as a first step; now that they are not starting from a microbial source, the technology can be developed in preclinical cell culture models to show efficacy and in mice to show in vivo activity.

And Dickinson also sees a bigger picture: "I am most excited by imagining what the post-CRISPR world looks like." He predicts that CRISPR will be successful in curing monogenic diseases, but new approaches are required for diseases that involve multiple genes in which "you need to inhibit one thing and increase the information flow through a series of other things-this synergy gives you the therapeutic outcome." For Dickinson, one avenue toward treating these kinds of disease lies in targeting gene expression at the RNA level.

Nicole Rusk

Published online: 30 July 2019

https://doi.org/10.1038/s41592-019-0526-4

Research papers

Rauch, S. et al. Programmable RNA-guided RNA effector proteins built from human parts. Cell 178, 122-134 (2019).
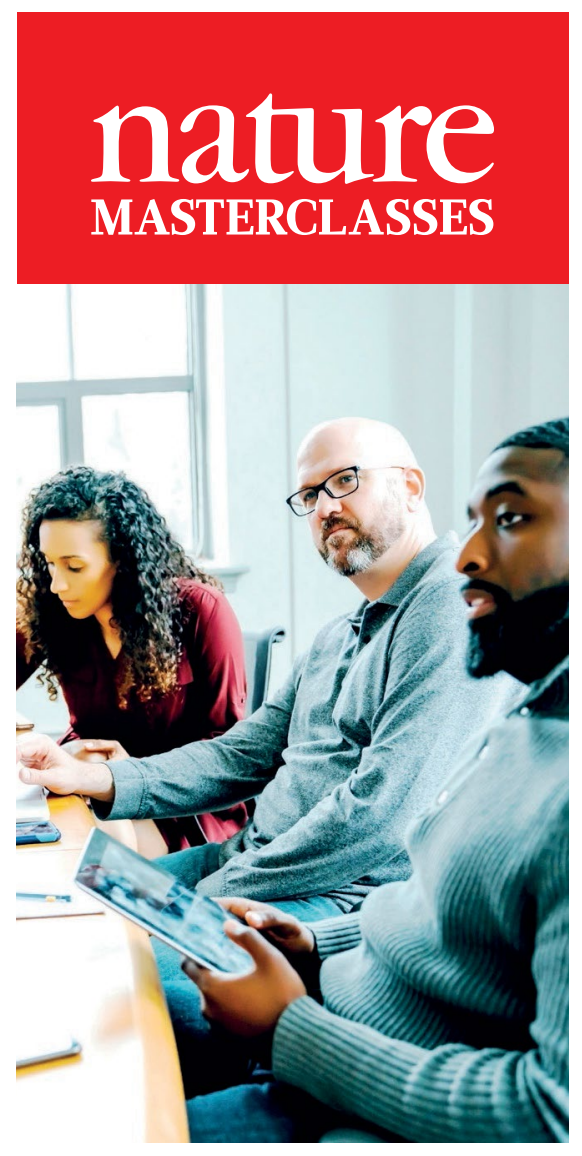

Workshops in

Scientific Writing

and Publishing

Delivered by Nature Research

journal editors and hosted

at institutions worldwide,

researchers learn how to

turn great science into great

papers.

Find out more at

masterclasses.nature.com

W masterclasses.nature.com

in Follow us on Linkedln 